spectral width of about $2 \Delta v \approx 25 \mathrm{GHz}$ is required, the electrical power spectrum density is $-136 \mathrm{dBm} / \mathrm{Hz}$ which is still $40 \mathrm{~dB}$ above the thermal noise limit.

As an example Fig. 2 shows the noise spectrum for unity $P_{\mathrm{el}}$ and $\Delta v=1 \mathrm{GHz}$. The solid line corresponds to eqn. 4 and the dashed line is the exact power spectrum accounting for the spectral width of the laser diode (presumed here to be $\Delta f=50 \mathrm{MHz}$ ). It can be seen in Fig. 2 that the spectrum of eqn. 4 is in good agreement with the actual power spectrum for frequencies $<2 \Delta v$. The evaluation of the power spectrum should therefore be limited to this frequency range.

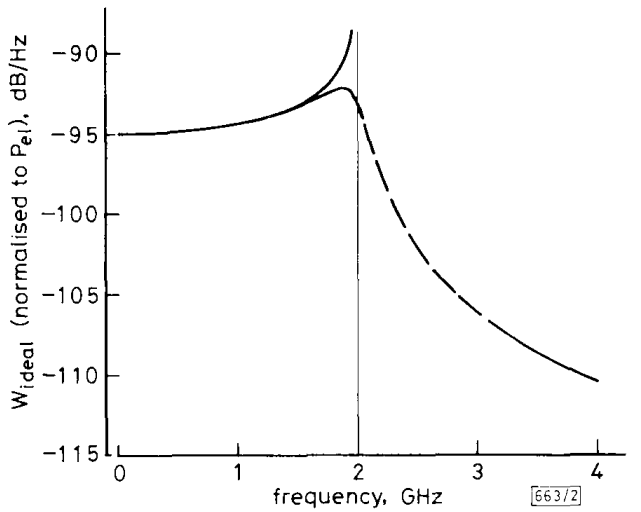

Fig. 2 Power density spectrum for ideal photodetector with unity total power and $\Delta v=1 \mathrm{GHz}$

- laser diode with $\Delta f=0$

- - - - laser diode with $\Delta f=50 \mathrm{MHz}$

Finally the power transfer function $H(f)$ of the photoreceiver can be expressed as

$$
|H(f)|^{2}=\frac{W_{m}(f)}{W_{\text {ideal }}(f)}
$$

where $W_{m}(f)$ is the measured power spectrum.

Experiment: The proposed method was verified by a preliminary experiment. Owing to the equipment used in the experiment, only the frequency range up to $1.8 \mathrm{GHz}$ was analysed. A DFB laser diode has been used with a current frequency transfer factor of about $0.25 \mathrm{GHz} / \mathrm{mA}$ at $100 \mathrm{kHz}$. For a $2 \mathrm{GHz}$-wide spectrum $(\Delta v=1 \mathrm{GHz})$ a current modulation amplitude of about $4 \mathrm{~mA}$ is thus required. Fig. 3 shows a set of results for the frequency response of the photoreceiver under test for different modulation indices $m$. For large $m$, large frequency modulations $\Delta v$ and wide measurement ranges are obtained, as shown in Fig. 3. The results show very good agreement for all modulation indices $m$, which demonstrates the good measurement reproducibility of this method.

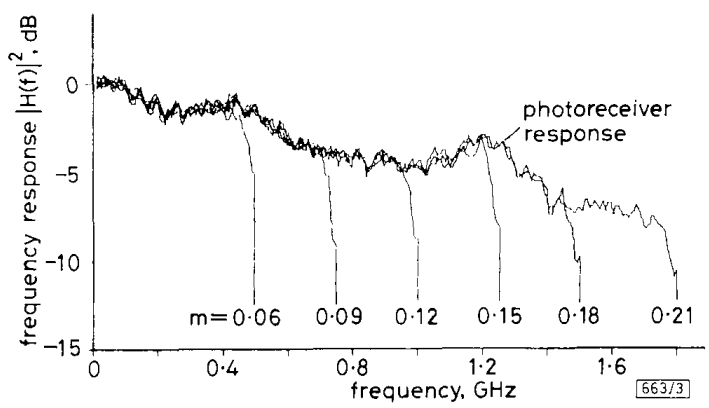

Fig. 3 Frequency response of photoreceiver tested

Conclusions: We have demonstrated a new technique for measuring the frequency response of a photoreceiver using a delayed self-homodyne set-up. This measurement technique has the advantage of a simple implementation. Even for measuring bandwidths of tens of $\mathrm{GHz}$, the power spectral density at the photoreceiver is about $40 \mathrm{~dB}$ above the thermal noise limit yielding reliable results. The measurement range is ultimately limited by the maximum frequency modulation of the laser diode. A wide frequency range of $\Delta v=10-100 \mathrm{GHz}$ would be possible, if tunable lasers ${ }^{10}$ are used.

J. WANO

17th March 1989

U. KRUGER

B. SCHWARZ

K. PETERMANN

Institut für Hochfrequenztechnik

Technische Universität Berlin

Einsteinufer 25, D-1000 Berlin 10, Federal Republic of German

References

1 BURRUS, C. A., BOWERS, J. E., and TUCKER, R. S.: 'Improved veryhigh-speed packaged InGaAs PIN punch-through photodiode' Electron. Lett., 1985, 21, pp. 262-263

2 SCHIMPE, R., BOWERS, J. E., and KOCH, T. L.: 'Characterisation of frequency response of $1.5 \mu \mathrm{m}$ InGaAsP DFB laser diode and InGaAs PIN photodiode by heterodyne measurement technique', Electron. Lett., 1986, 22, pp. $453-454$

3 PICCARI, L., and SPANO. P.: 'New method for measuring ultrawide frequency response of optical detectors', Electron. Lett., 1982, 18, pp. 116-118

4 eicheN, E., and Silletti, A.: 'Bandwidth measurements of ultrahigh-frequency optical detectors using the interferometric FM sideband technique', J. Lightwave Technol., 1987, LT-5, pp. 13771381

5 HEMERY, E., CHUSSEAU, L., and LOURTIOZ, J.-M.: 'Frequency characterisation of photodetectors by Fabry-Perot interferometry of modulated semiconductor lasers', Electron. Lett., 1989, 25, pp. $42-44$

6 ANDERSSON, T., JOHNSTON, A, R. and EKLUND, H. 'Temporal and frequency response of avalanche photodiodes from noise measurements', Appl. Opt., 1980, 19, pp. 3496-3499

$7 \mathrm{RYU}$, S., and Yamamoto, S.: Measurement of direct frequency modulation characteristics of DFB-LD by delayed self-homodyne technique', Electron. Lett., 1986, 22, pp. 1052-1054

8 PETERMANN, $K$. 'Laser diode modulation and noise' (Kluwer Academic Publ., Dordrecht, The Netherlands, 1988)

9 SCHWARZ, B.: 'Messung des Frequenzganges eines optischen Empfängers'. Studienarbeit, Institut für Hochfrequenztechnik, Technische Universität Berlin, 1988

10 YoshikUni, Y., and Motosugi, G.: 'Multielectrode distributed feedback laser for pure frequency modulation and chirping suppressed amplitude modulation', J. Lightwave Technol., 1987, LT-5, pp. $516-52$

\section{NEW RADIX-2-BASED ALGORITHM FOR FAST MEDIAN FILTERING}

Indexing terms: Signal processing, Alyorithms, Filters, Image processing

A fast radix-2-based median filtering algorithm is proposed The median is determined bit-by-bit successively by eliminating the samples whose previous bits are different to that of the median. The intermediate computations of the algorithm do not involve any array computation, nor any memory. The worst-case computational complexity of the algorithm is $O(w)$ for $w$ samples.

Introduction: Median filtering is a nonlinear smoothing technique used in signal and image processing to filter out the impulsive noises while preserving the edge-information. In the applications of median filtering, a window of size $w$ moves over the sampled values of the signal or image, and then the median of the samples within the window is computed and written as the output pixel at the location of the centre of the window. ${ }^{1}$

Various median filtering algorithms for software and for hardware implementations have been published. ${ }^{2-7}$ A comparison of the computational requirements of various 
software-based median filtering algorithms is given by Ataman et al., ${ }^{4}$ and it is claimed that their algorithm is more efficient than the others. More recently, V. Rao and S. Rao ${ }^{6}$ have presented a software-based median filtering algorithm which requires less processing than Ataman's algorithm.

However, in both Ataman's ${ }^{4}$ and Rao's ${ }^{6}$ algorithms, an intermediate array is computed and updated. This requires too much computation. Since some of the elements of these arrays are not used in the median computation, there is some redundancy. Furthermore, the arrays of Ataman's and Rao's algorithms require $2^{r}$ bytes of memory.

In this letter, an alternative fast median computation procedure is proposed. The computation of full-word median requires $r$ full-word comparisons and a number of bit-level operations proportional to $w \times r$ where $w$ is the window size and $r$ is the word-length. The proposed algorithm does not require any intermediate array computation, any memory except the storage of the window samples and a few registers, any histogram computation, nor any data sorting. Furthermore, the algorithm can be easily mapped to hardware either as a single chip or as a small-sized printed circuit board.

Algorithm: Let $\left\{x_{1}, x_{2}, x_{3}, \ldots, x_{w}\right\}, w$ is odd, be the set of samples in the window. Let $\left\{u_{i 1}, u_{i 2}, u_{i 3}, \ldots, u_{i r}\right\}$ and $\left\{m_{1}, m_{2}\right.$, $\left.m_{3}, \ldots, m_{r}\right\}$ be the radix -2 representations of the $x_{i}$ and median, respectively. In other words, $u_{i j}$ is the $j$ th most significant bit of $i$ th input sample, and $m_{i}$ is the $i$ th most significant bit of the median.

Let $t=(\omega+1) / 2$. Then the median is the $t$ th smallest sample in the window. Thus the median search task is to find the $t$ th smallest sample. The algorithm finds this sample successively bit-by-bit starting from the most significant bit. To do that, first we start counting the $0 \mathrm{~s}$ in the most significant bits of the samples, and call that number $Z_{1}$. If $Z_{1} \geq t$ then the first bit of the median is set to $0\left(m_{1}=0\right)$, and $t$ stays as it is. If $Z_{1}<t$ then $m_{1}=1$ and $t$ is updated as $t \leftarrow t-Z_{1}$. In the second step, $Z_{2}$ is found by counting $0 \mathrm{~s}$ in the second most significant bits of the samples whose most significant bits are the same as $m_{1}$, and repeating the procedure of the first step. Consequently, in the $k$ th step, $Z_{k}$ is obtained by counting $0 \mathrm{~s}$ in the $k$ th bits of the samples whose first $(k-1)$ bits are the same as that of the median.

To specify the samples whose first $k$ bits are the same as that of the median, a flag is assigned to each sample. This flag can be checked and updated during the computations as follows: let $c_{i}$ be the flag for the sample $x_{i}$, if $c_{i}=1$ at $k$ th step, then the sample $x_{i}$ is enabled in the computation of $Z_{k}$; otherwise it is disabled (in the computation of $Z_{k}$, only enabled samples are considered). At the $k$ th step, each $c_{i}$ is updated in such a way that if $c_{j}=1$ and $u_{j k} \neq m_{k}$ then $c_{j}=0$, else it remains as it is, $c_{j}=1$. Note that if a flag takes the value 0 at any time, then it never changes.

Let the number of enabled samples be denoted by $S_{c}$ which is equal to the sum of $c_{i} s$. Then, during the computation of the median when $S_{c}=t=1$ the algorithm terminates. In this case, since there is only one enabled sample, the undetermined bits of the median are the same as that of this sample.

The complete procedure of the algorithm is as follows:

Step 1: Set $k=1, t=(w+1) / 2$, and $c_{i}=1$ for $1 \leq i \leq w$

Step 2: Compute $Z_{k}$

Step 3: If $Z_{k}<t$, then $m_{k}=1$ and $t \leftarrow t-Z_{k}$, else $m_{k}=0$

Step 4: Update $c_{i}$ s and find $S_{c}=\sum_{i=1}^{w} c_{i}$

Step 5: If $S_{c}=t=1$, then set $m_{j}=u_{i j}$ whose $c_{i}=1$ and $k \leq j \leq r$, and go to Step 8

Step 6: $k \leftarrow k+1$

Step 7: If $k=r+1$ go to Step 8, else go to Step 2

Step 8: Stop

As an example, consider the set of samples $\{5,4,11,1,14,7$, $9,5,10\}$. Presume that each of these samples is a 4-bit number (i.e. $r=4$ ). The procedure to find the median of this set is as follows :

$\begin{array}{llllllllll}x_{i} & 5 & 4 & 11 & 1 & 14 & 7 & 9 & 5 & 10 \\ u_{i 1} & 0 & 0 & 1 & 0 & 1 & 0 & 1 & 0 & 1 \\ u_{i 2} & 1 & 1 & 0 & 0 & 1 & 1 & 0 & 1 & 0 \\ u_{i 3} & 0 & 0 & 1 & 0 & 1 & 1 & 0 & 0 & 1 \\ u_{i 4} & 1 & 0 & 1 & 1 & 0 & 1 & 1 & 1 & 0 \\ k=1 & & k=2 & & k=3 \\ \left.k c_{i}\right\}: 111111111 & \left\{c_{i}\right\}: 110101010 & \left\{c_{i}\right\}: 110001010 \\ t=5 & & t=5 & & t=4 \\ Z_{i}=5 & & Z_{2}=1 & & Z_{3}=3 \\ Z_{1} \geq t & & Z_{2}<t & & Z_{3}<t \\ m_{1}=0 & & m_{2}=1 & & m_{3}=1 \\ t t=t) & & t=5-1=4 & t=4-3=1 \\ \left\{c_{i}\right\}: 110101010 & \left\{c_{i}\right\}: 110001010 & \left\{c_{i}\right\}: 000001000 \\ S_{c}=5 & & S_{c}=4 & & S_{c}=1 \\ S_{c} \neq t \neq 1 & & S_{c} \neq t \neq 1 & S_{c}=t=1 \\ k=1+1=2 & & k=2+1=3 & m_{4}=1\end{array}$

Thus, the median represented by $\left\{m_{1}, m_{2}, m_{3}, m_{4}\right\}$ is found to be $\{0,1,1,1\}$. This is the radix-2 representation of 7 which can also be found by sorting the samples and taking the middle sample.

Concluding remarks: A fast radix-2-based median filtering algorithm with worst-case computational complexity $\mathrm{O}(w \times r)$ is presented. All operations of the algorithm are performed recursively as the bits arrive, therefore there is no redundant computation. Since most of the operations are in bit-level, the algorithm is very efficient if it is implemented in a low-level programming language. For the real-time applications, since one has to consider the worst-case computational complexity, the algorithm proposed here is more efficient than recently proposed algorithms. ${ }^{4,6}$ In addition, the algorithm does not require any memory except storage of the window samples and a few registers, whereas the other two algorithms require $2^{r}$ bytes of memory. Furthermore, for hardware implementation, the proposed algorithm requires considerably less circuitry than the others. A fast median filter unit can be built using VLSI techniques, or even using conventional components on a small printed circuit board. In any case, the cost of the hardware implementation grows linearly with both the window size $w$ and the word-length $r$.

\section{KARAMAN}

L. ONURAL

13th January 1989

Department of Electrical \& Electronics Engineering

Bilkent University

PO Box 8,06572 Maltepe, Ankara, Turkey

\section{References}

1 Gallagher, N. C., JUN., and WISE, G. L.: 'A theoretical analysis of the properties of median filters', IEEE Trans. Acoust. Speech Signal Process., 1981, ASSP-29, pp. 1136-1141

2 GARIBOTTO, G., and LAMBARELLI, L.: 'Fast on-line implementation of two-dimensional median filtering', Electron. Lett., 1979, 15, pp. $24-25$

3 HUANG, T. S., YANG, G. J., and TANG, G. Y.: 'A fast two-dimensional median filtering algorithm', IEEE Trans. Acoust. Speech Signal Process., 1979, ASSP-27, pp. 13-18

4 ataman, E., AATRE, v. K., and wong, K. M.: 'A fast method for real-time median filtering', IEEE Trans. Acoust. Speech Signal Process., 1980, ASSP-28, pp. 415-421

5 OFLAZER, K.: 'Design and implementation of a single-chip 1-D median filter', IEEE Trans. Acoust. Speech Signal Process., 1983, ASSP-31, pp. 1164-1168

6 RAO, v. v. B., and RAO, K. S.: 'A new algorithm for real-time median filtering', IEEE Trans. Acoust. Speech Signal Process., 1986, ASSP34, pp. 1674-1675

7 Karaman, M., ONURAL, L., and ATalar, A.: 'Design and implementation of a general purpose median filter in VLSI, in BRODERSEN, R. W., and MOSCOVITZ, H. S.: 'VLSI Signal Processing III' (IEEE Press, NY, 1988), pp. 111-119

ELECTRONICS LETTERS 25th May 1989 Vol. 25 No. 11 